\title{
Physicochemical and Morphological Characterizations of Glyceryl Tristearate/Castor Oil Nanocarriers Prepared by the Solvent Diffusion Method
}

\author{
Cristiana L. Dora, ${ }^{a}$ Jean-Luc Putaux, ${ }^{b}$ Isabelle Pignot-Paintrand, ${ }^{b}$ Frédéric Dubreuil, ${ }^{b}$ \\ Valdir Soldi, ${ }^{c}$ Redouane Borsali ${ }^{b}$ and Elenara Lemos-Senna*,a \\ ${ }^{a}$ Laboratório de Farmacotécnica, Departamento de Ciências Farmacêuticas, Centro de Ciências da Saúde, \\ Campus Universitário, Trindade, 88040-970 Florianópolis-SC, Brazil \\ ${ }^{b}$ Centre de Recherches sur les Macromolécules Végétales (CERMAV-CNRS)," \\ BP 53, F38041 Grenoble Cedex 9, France \\ 'Departamento de Química, Centro de Ciências Físicas e Matemáticas, Universidade Federal de Santa Catarina, \\ Campus Universitário, Trindade, 88040-970 Florianópolis-SC, Brazil
}

Neste estudo, nanocarreadores lipídicos foram preparados a partir do triestearato de glicerila e óleo de rícino em diferentes proporções pela técnica de difusão do solvente a quente. Estearato de PEG-660 ou Poloxamer 188 e lecitina de soja foram usados como surfactante e cossurfactante, respectivamente. Características estruturais e físico-químicas das partículas foram investigadas por meio de espalhamento de luz dinâmico (DLS), calorimetria exploratória diferencial (DSC), espalhamento de raios X a altos ângulos (WAXS), microscopia de força atômica (AFM) e microscopia eletrônica de transmissão (TEM e cryo-TEM). A análise por DLS indicou que partículas menores foram obtidas quando o lipídio líquido foi usado sozinho para obter as nanoemulsões (NEs). Técnicas microscópicas (TEM e cryo-TEM) mostraram que as NEs apresentaram formas esféricas, enquanto as nanopartículas lipídicas sólidas (NLSs) e os carreadores lipídicos nanoestruturados (CLNs) apresentaram formas mais complexas. As NLSs exibiram estrutura cristalina lamelar, enquanto os CLNs exibiram uma estrutura mista composta de fase cristalina lamelar em contato com compartimento oleoso. Estes experimentos contribuem para um melhor entendimento da capacidade de incorporação e liberação de fármacos destes nanocarreadores.

In this study, drug delivery nanocarriers based on glyceryl tristearate and castor oil at different ratios were prepared by the hot solvent diffusion method. PEG 660-stearate or Poloxamer 188 and soybean lecithin were used as surfactant and co-surfactant, respectively. Structural and physicochemical characteristics of the particles were investigated by means of dynamic light scattering (DLS), differential scanning calorimetry (DSC), wide-angle X-ray scattering (WAXS), atomic force microscopy (AFM) and transmission electron microscopy (TEM and cryo-TEM). DLS data indicated that smaller particles were obtained when the liquid lipid was used alone to prepare nanoemulsions (NEs). TEM and cryo-TEM images showed that NEs presented spherical particles, whereas solid lipid nanoparticles (SLNs) and nanostructured lipid carriers (NLCs) presented more complex shapes. SLNs exhibited lamellar crystal structure, while NLCs displayed a hybrid structure composed of a lamellar crystalline phase in contact with a liquid oil compartment. These experiments contribute to a better understanding of the structure and release performance of these drug carrier systems.

Keywords: solid lipid nanoparticles, nanostructured lipid carriers, nanoemulsions, structural investigation, drug delivery systems

*e-mail: lemos@ccs.ufsc.br

"Affiliated with Université Joseph Fourier and member of Institut de

Chimie Moléculaire de Grenoble and Institut Carnot PolyNat, France 


\section{Introduction}

In the last decade, lipid-based colloidal carriers have emerged as a way to deliver poorly water-soluble drugs, especially when oral delivery is required. Colloidal carriers can consist of solid or liquid lipids or a mixture of both in various ratios and display a wide range of particle sizes. The advantages of lipid-based formulations include a wide spectrum of applications, the use of biodegradable physiological lipids recognized as safe or having a regulatory acceptance status, and the possibility of scaling up to an industrial production level. ${ }^{1-3}$ Lipid-based colloidal carriers have also demonstrated a great ability to enhance the oral absorption of lipophilic drugs, the mechanisms of which encompass lipid digestion by components of the gastrointestinal tract and selective uptake by the lymphatic system. ${ }^{4}$

In previous studies, our group prepared lipid-based colloidal dispersions via hot solvent diffusion method with the aim of carrying quercetin (QU), a lipophilic natural drug that displays antitumor, anti-inflammatory and antioxidant activities but has a very low oral bioavailability. In particular, castor oil and tristearin were employed to obtain oil-in-water nanosized emulsions (NEs) and solid lipid nanoparticles (SLNs), respectively. Moreover, nanostructured lipid carriers (NLCs) were prepared by mixing both solid and liquid lipids in different ratios. For the lipid formulations assayed, poly(ethylene glycol) (660)-12-hydroxystearate (PEG 660-stearate, Solutol HS15 ${ }^{\circledR}$ ) or Poloxamer 188 (Lutrol F68 ${ }^{\circledR}$ ) were used as hydrophilic surfactants, and hydrogenated soybean lecithin was used as a lipophilic surfactant. The results demonstrated that the quercetin loading increased with increasing castor oil concentration in the total lipid mixture. ${ }^{5}$ However, the way in which the drug loading was affected by the structural properties of the particles still needs to be determined. It has been recognized that the composition of a lipid formulation influences the shape and structure of the particles, which may or may not display phase separation. ${ }^{3}$

The lipid materials used to prepare matrix nanocarriers usually occur in different allomorphs and, since the hot solvent diffusion method used to prepare colloidal suspensions includes a thermal procedure and a recrystallization step, polymorphism is an important issue that needs to be investigated. In particular, tristearin crystallizes into four allomorphs $\left(\gamma, \alpha, \beta^{\prime}\right.$ and $\left.\beta\right)$, and their formation depends on the preparation conditions, i.e., cooling and stirring rates. ${ }^{6}$ Formulation composition has also been shown to affect the crystal structure of the particles. For instance, the use of unmodified soybean lecithin leads to the formation of $\beta$-tristearin during the preparation of SLNs, while the use of hydrogenated fully saturated soybean lecithin results in a predominant fraction of the metastable $\alpha$-form. ${ }^{7}$

Therefore, in the present study, the structural and physicochemical characteristics of lipid-based nanocarriers prepared from tristearin and castor oil using a hot solvent diffusion method were investigated. Complementary analytical techniques, including dynamic light scattering (DLS), differential scanning calorimetry (DSC), wide-angle X-ray scattering (WAXS), atomic force microscopy (AFM) and transmission electron microscopy (TEM and cryo-TEM) were used to provide detailed information on the morphology, structure and properties of the colloidal lipid particles.

\section{Experimental}

Materials

Castor oil (CO), a triglyceride in which approximately $90 \%$ of the fatty acid chains are ricinoleic acid, was purchased from Natural Pharma (São Paulo, Brazil). Hydrogenated soybean lecithin (Lipoid S75-3N) was purchased from SP Pharma (São Paulo, Brazil). According to the manufacturer, the product contains from 67 to 73\% phosphatidyl choline and lysophosphatidyl choline. Tristearin (Dynasan 118, TS) was kindly donated by Sasol (Louisiana, USA). Fatty acid fraction was $99 \%$ pure for this triglyceride quality and the hydroxyl value was below 5. Polyoxypropylene-polyoxyethylene block copolymer (Lutrol F-68 NF ${ }^{\circledR}$, called Poloxamer 188 in this work) and polyethyleneglycol mono- and diesters of 12-hydroxystearic acid (Solutol HS-15 ${ }^{\circledR}$, called PEG 660-stearate in this work) were kindly donated by BASF (Trostberg, Germany). Ethanol, acetone and other chemicals used were analytical grade.

\section{Preparation of the lipid-based nanocarriers}

Lipid-based nanocarriers were prepared by a hot solvent diffusion method. ${ }^{8,9}$ Briefly, $20 \mathrm{mg}$ of lecithin and $100 \mathrm{mg}$ of TS, CO or their mixture (1:1) were completely dissolved into an acetone/ethanol $(60: 40 \mathrm{v} / \mathrm{v})$ mixture at $60{ }^{\circ} \mathrm{C}$. The resulting organic solution was quickly poured into $50 \mathrm{~mL}$ of an aqueous solution containing $0.1 \%(\mathrm{w} / \mathrm{v})$ PEG 660-stearate or 1\% (w/v) Poloxamer 188 maintained under magnetic stirring at $82{ }^{\circ} \mathrm{C}$. The resulting colloidal suspensions were then cooled to room temperature. The organic solvents were evaporated under reduced pressure, and the final volumes were adjusted to $20 \mathrm{~mL}$. Finally, the colloidal suspensions were filtered through 
$8 \mu \mathrm{m}$ filter paper in order to remove any environmental contaminant inadvertently introduced in the colloidal suspensions during the nanoparticle preparation. For the DSC and WAXS studies, SLN and NLC colloidal suspensions were freeze-dried. The final compositions of the lipid-based colloidal suspensions are shown in Table 1.

\section{Particle size analysis}

The particle sizes and polydispersity indices (PDI) of the colloidal suspensions were determined by DLS using an ALV 5000 (ALV, Germany) and a Zetasizer Nano Series (Malvern Instruments, Worcestershire, UK). The samples were previously diluted in ultrapure Milli- $\mathrm{Q}^{\circledR}$ water. Each size analysis lasted $300 \mathrm{~s}$ at $25^{\circ} \mathrm{C}$. The analysis was performed at a scattering angle of $90^{\circ}$ or $173^{\circ}$, depending on which equipment was used (ALV 5000 or Zetasizer, respectively). The hydrodynamic radius $\left(\mathrm{R}_{\mathrm{h}}\right)$ was determined using the Stokes-Einstein equation, $\mathrm{R}_{\mathrm{h}}=\left(\mathrm{k}_{\mathrm{B}} \mathrm{T} / 6 \pi \eta \mathrm{D}\right)$, in which $\mathrm{k}_{\mathrm{B}}$ is the Boltzmann constant, $\mathrm{T}$ is the temperature, $\mathrm{D}$ is the diffusion coefficient and $\eta$ is the viscosity of the medium $\left(\eta=0.89 \mathrm{mPa}\right.$ s at $\left.25^{\circ} \mathrm{C}\right)$. The mean particle size was approximated as the $\mathrm{z}$-average diameter. The PDI value was taken as the width of the corresponding distribution, calculated by the cumulant method, assuming a spherical shape.

\section{Transmission electron microscopy (TEM and cryo-TEM)}

Drops of $0.001 \%(\mathrm{w} / \mathrm{v})$ suspensions were deposited on carbon-coated copper grids and negatively stained with $2 \%(w / v)$ uranyl acetate. Specimens for cryo-TEM were prepared by quench-freezing thin liquid films of the suspensions in liquefied ethane $\left(-171^{\circ} \mathrm{C}\right)$. These specimens were mounted on a liquid-nitrogen-cooled Gatan 626 cryoholder and transferred to the microscope. The specimens were observed at low temperature $\left(-180{ }^{\circ} \mathrm{C}\right)$ using a Philips CM200 cryoelectron microscope
(FEI Company, Eindhoven, The Netherlands) operating at $80 \mathrm{kV}$. A low-dose procedure was used to reduce radiation damage in the areas of interest. The images were recorded on Kodak SO163 films.

\section{Atomic force microscopy (AFM)}

Drops of dilute lipid-based nanocarrier suspensions were deposited onto freshly cleaved mica. After $20 \mathrm{~min}$, the samples were dried with nitrogen. AFM experiments were performed on a Molecular Imaging PicoPlus instrument. Topographic images were recorded in tapping mode.

\section{Wide-angle X-ray scattering (WAXS)}

WAXS studies were carried out using freeze-dried or concentrated colloidal suspensions. The powders were sealed between two Mylar foils, whereas the liquids were poured into glass capillaries ( $0.7 \mathrm{~mm}$ outer diameter) and flame-sealed. Both types of specimens were mounted on $0.2 \mathrm{~mm}$ collimators and subjected to Ni-filtered $\mathrm{CuK}_{\alpha}$ radiation $(\lambda=1.543 \mathrm{~nm})$ using a Philips PW3830 generator operating at $30 \mathrm{kV}$ and $20 \mathrm{~mA}$. WAXS powder patterns were recorded on Fujifilm imaging plates, and read with a Fujifilm BAS-1800II Bio-imaging analyzer. Diffraction profiles were obtained by radially integrating the intensity over the whole spectra. The diffraction rings were calibrated using a $\mathrm{CaCO}_{3}$ standard.

\section{Differential scanning calorimetry}

DSC analyses were performed using a TA Q200 instrument. About 2-20 mg of freeze-dried colloidal suspensions or the bulk materials used to prepare the lipid nanocarriers were accurately weighted into standard aluminum pans, which were tightly sealed. The samples were cooled to $-20^{\circ} \mathrm{C}$ and then heated to $100^{\circ} \mathrm{C}$ at a scan rate of $10^{\circ} \mathrm{C} \mathrm{min}^{-1}$. An empty pan was used as a reference.

Table 1. Composition of the lipid-based colloidal suspensions

\begin{tabular}{|c|c|c|c|c|c|c|}
\hline & \multicolumn{3}{|c|}{ Poloxamer 188} & \multicolumn{3}{|c|}{ PEG 660-stearate } \\
\hline & SLN & NLC & $\mathrm{NE}$ & SLN & NLC & $\mathrm{NE}$ \\
\hline Tristearin / mg & 100 & 50 & - & 100 & 50 & - \\
\hline Castor oil / mg & - & 50 & 100 & - & 50 & 100 \\
\hline Lecithin / mg & 20 & 20 & 20 & 20 & 20 & 20 \\
\hline Poloxamer $188 /$ wt. $\%^{\text {a }}$ & 2.5 & 2.5 & 2.5 & - & - & - \\
\hline PEG 660-stearate / wt. $\%^{\mathrm{a}}$ & - & - & - & 0.25 & 0.25 & 0.25 \\
\hline Water q.s. to / mL & 20 & 20 & 20 & 20 & 20 & 20 \\
\hline
\end{tabular}

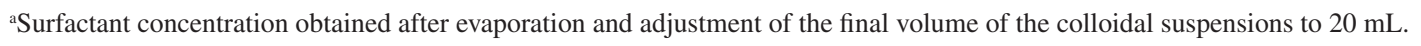




\section{Results}

\section{Particle size}

In the present study, the lipid-based nanocarriers were prepared using the solvent diffusion method. This method consists in forming a hot oil-in-water emulsion after addition of a water-miscible organic solution containing the lipid and a lipophilic surfactant into an aqueous solution having a hydrophilic surfactant. Turbulence at the interface of the emulsion droplets (Marangoni effect) is produced by the very rapid diffusion rate of the water-miscible organic solvent into the aqueous phase, resulting in the spontaneous formation of submicronic droplets. ${ }^{8,9}$ All formulations were prepared at $82{ }^{\circ} \mathrm{C}$ to ensure that the lipid (tristearin) remained liquid. After cooling, the resulting colloidal dispersions were macroscopically homogeneous and had a milky appearance, regardless of the type of lipid.

The mean particle size and particle size distribution of the nanocarriers as determined by DLS are listed in Table 2. The dynamic light scattering correlation functions and the corresponding hydrodynamic radii $\left(\mathrm{R}_{\mathrm{h}}\right)$ of the colloidal dispersions obtained using a single $90^{\circ}$ scattering angle are shown in Figure 1. All colloidal dispersions displayed polydispersity indices lower than 0.3 for different angles, suggesting that monodisperse populations of particles were obtained. However, the mean particle sizes were around 340, 200 and $140 \mathrm{~nm}$ for SLNs, NLCs and NEs, respectively, when they were measured in the ALV 500 at an angle of $90^{\circ}$, whereas they were 250, 170 and $140 \mathrm{~nm}$ for SLNs, NLCs and NEs, respectively, when measured in the Zetasizer at an angle of $173^{\circ}$. The nature of the surfactant did not seem to have any effect on the particle size, but the nature of the lipid (solid, liquid or the mixture) did. When only the castor oil was used, the particle size and the PDI value were lower.

\section{Particle morphology}

TEM images of the NE suspensions prepared with $\mathrm{CO}$ as the lipid component and in the presence of
Poloxamer 188 or PEG 660-stearate are shown in Figures $2 \mathrm{a}$ and $2 \mathrm{~b}$. The dark outline seen on the bigger particles is due to the fact that the film of the dry uranyl acetate was rather thick and only the top part of the spherical particle protruded out of the stain.

For a given formulation, the size distributions of the negatively stained and ice-embedded particles were quite similar, suggesting that the particles did not significantly deform during drying on the carbon films. Some limited particle deformation could be seen in the cryo-TEM images due to the confining effect inside the thin film of vitreous ice (Figures $2 \mathrm{c}$ and $2 \mathrm{~d}$ ).

When only TS was used as the lipid component to prepare SLNs, lamellar crystals with irregular and angular shapes were observed (Figures $3 a$ and $3 b$ ). No satisfying image of the lamellae could be recorded by cryo-TEM. The thin crystals were systematically oriented perpendicular to the electron beam, thus generating very poor amplitude contrast. The crystals also proved to degrade extremely fast under electron illumination.

The thickness of the lamellar SLNs was estimated by AFM imaging. Figures $4 \mathrm{a}$ and $4 \mathrm{~b}$ are tapping-mode images of SLNs prepared with Poloxamer 188 and PEG 660-stearate, respectively.

For the first sample, height profiles were taken longitudinally across objects that appeared to be multilamellar (Figure 4a). The stepped aspect of the profiles suggests that the particles are made of irregularly stacked lipid layers with a minimum increment of about 4-5 $\mathrm{nm}$. Considering the structure of $\beta$-TS, this would correspond to the spacing between two lipid layers, i.e., $4.5 \mathrm{~nm} .^{7} \mathrm{In}$ Figure $4 \mathrm{a}$, particle 1 thus consists of a first group of four lipid layers (about $15 \mathrm{~nm}$ ) with two additional smaller layers on top, each creating a step of about $5 \mathrm{~nm}$, for a total thickness of about $25 \mathrm{~nm}$. Particle 2 consists of a first group of three lipid layers (about $10 \mathrm{~nm}$ ) with an additional smaller layer on the top $(5 \mathrm{~nm})$ for a total thickness of about $15 \mathrm{~nm}$. The stepped aspect was less clearly defined for SLNs prepared with PEG 660-stearate (Figure 4b). Height profiles were taken from two particles. Average thicknesses of about 5 and $10 \mathrm{~nm}$ were measured from

Table 2. Mean particle size (nm) and polydispersity index (PDI) measured by DLS for the lipid-based colloidal suspensions

\begin{tabular}{|c|c|c|c|c|}
\hline & \multicolumn{2}{|c|}{ PEG 660 -stearate 0.25 wt. $\%$} & \multicolumn{2}{|c|}{ Poloxamer 1882.5 wt. $\%$} \\
\hline & Mean size ALV 5000 / $\mathrm{nm}^{\mathrm{a}}$ (PDI) & Mean size Zetasizer / $\mathrm{nm}^{\mathrm{b}}$ (PDI) & Mean size ALV 5000 / nm (PDI) & Mean size Zetasizer / $\mathrm{nm}^{\mathrm{b}}$ (PDI) \\
\hline SLN & $342(0.186)$ & $255(0.28)$ & $343(0.176)$ & $279(0.24)$ \\
\hline NLC & $221(0.110)$ & $160(0.09)$ & $215(0.153)$ & $184(0.08)$ \\
\hline $\mathrm{NE}$ & $150(0.123)$ & $130(0.14)$ & $142(0.137)$ & $156(0.09)$ \\
\hline
\end{tabular}

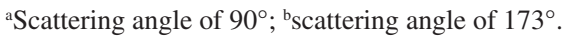



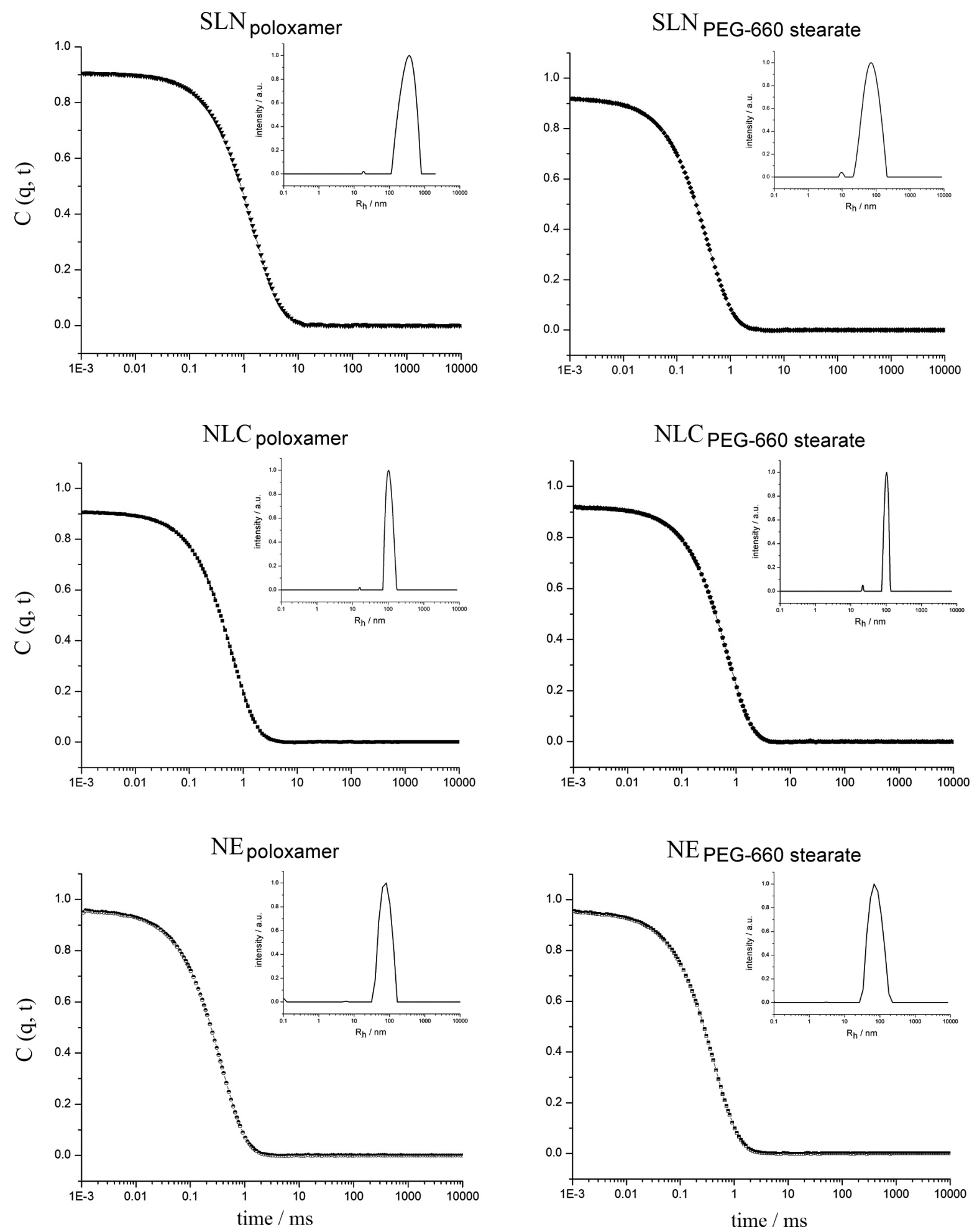

Figure 1. Correlation function and hydrodynamic radius $\left(\mathrm{R}_{\mathrm{h}}\right)$ obtained using an ALV-5000 at $90^{\circ}$ scattering angle for lipid-based nanocarriers containing 2.5 wt.\% Poloxamer 188 (left column) or 0.25 wt.\% PEG 660 stearate (right column).

particles 1 and 2, respectively. The $5 \mathrm{~nm}$ increment is in agreement with the value observed in SLNs prepared with Poloxamer 188. Particles 1 and 2 would thus be made of two and three lipid layers, respectively.
As seen in Figures 3c and 3d, negatively stained NLCs particles prepared by mixing $\mathrm{CO}$ with TS presented a clearly different morphology. Irregular 100-400 nm bilobular objects were mostly observed, surrounded by $10-50 \mathrm{~nm}$ 

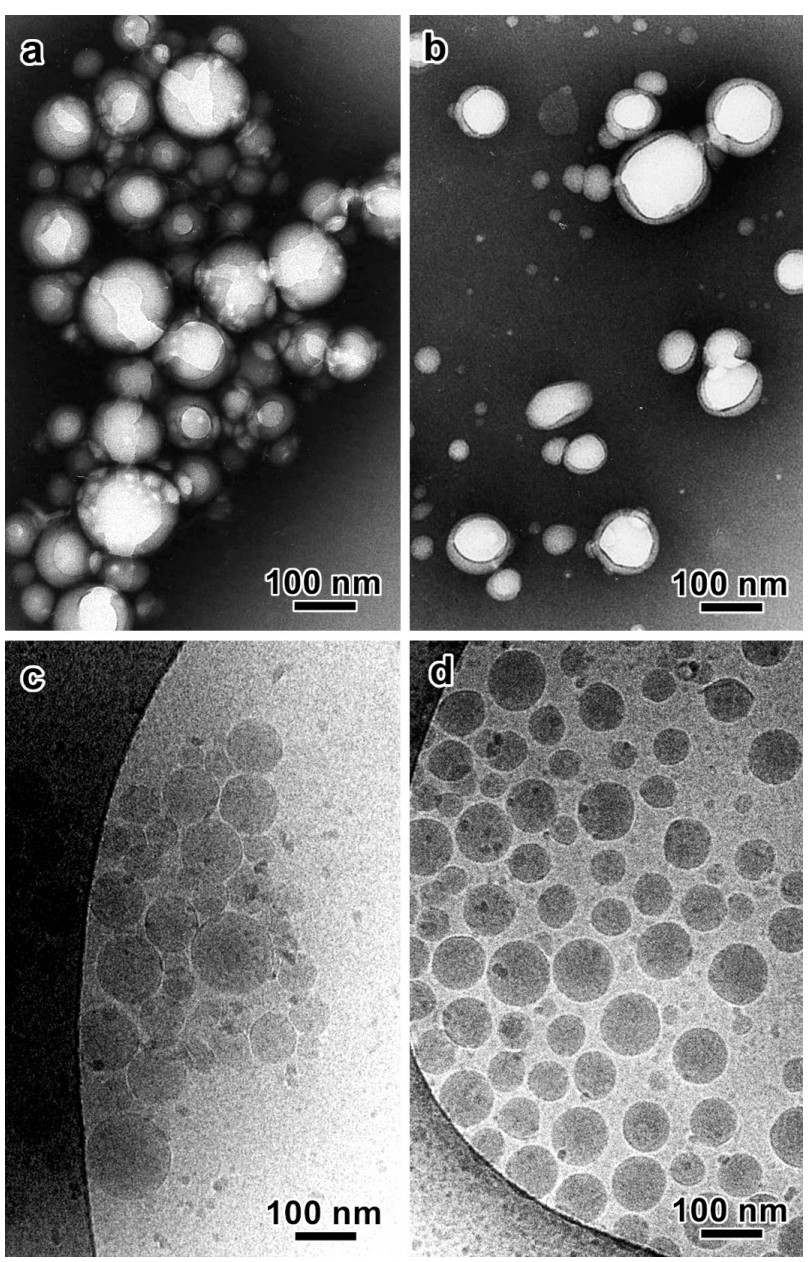

Figure 2. TEM images of NE nanocarriers prepared in the presence of Poloxamer 188 (a, c) and PEG 660-stearate (b, d) in the aqueous phase; $(\mathrm{a}, \mathrm{b})$ negative staining and $(\mathrm{c}, \mathrm{d})$ cryo-TEM.

particles. In particular, in the NLC sample prepared in the presence of PEG 660-stearate, 10-30 nm particle sizes were observed. They are probably micelles of pure PEG 660-stearate or swollen micelles with an oil-enriched core surrounded by a surfactant layer (Figure 3d). In both types of NLC preparation, one lobe of the larger particles is rather transparent, while the other is darker. Hypotheses can be made to account for this peculiar contrast, assuming that each lobe corresponds to the liquid and solid lipid phases: (i) each lobe has a different thickness, with the thickest one protruding farther out of the stain layer; or (ii) the stain preferentially interacts with one of the lobes (hence the darker contrast). Cryo-TEM images confirmed that NLC particles exhibited a variety of shapes and sizes (Figure 5).

Several NLC particles prepared in the presence of Poloxamer 188 are discoidal or spheroidal but parts of their peripheries are outlined by discontinuous dark fringes (Figure 5a). In these regions, the curvature is smaller and two dark fringes sometimes join to give a triangular shape to the particle. This higher rigidity and darker contrast is likely
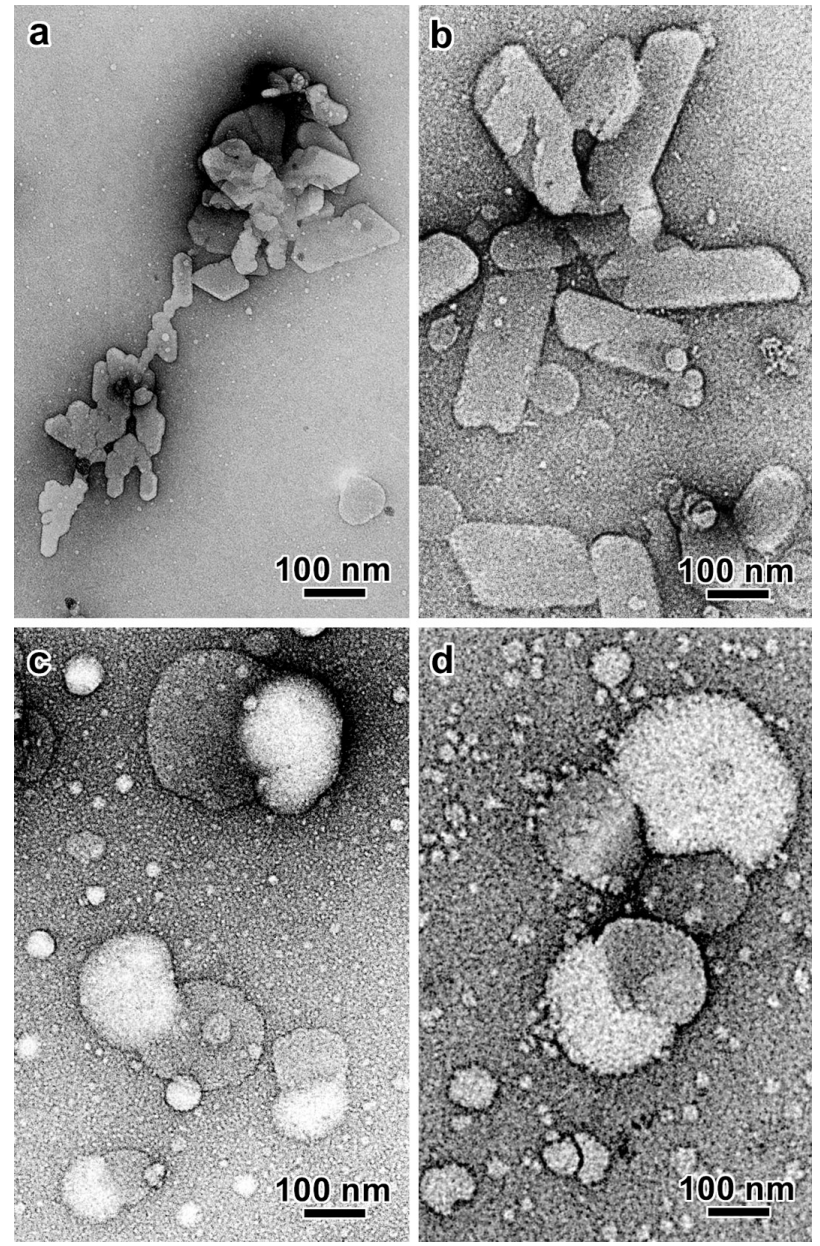

Figure 3. TEM images of negatively stained SLN (a, b) and NLC (c, d) particles prepared in the presence of Poloxamer $188(\mathrm{a}, \mathrm{c})$ and PEG 660-stearate $(b, d)$ in the aqueous phase.

related to the presence of a single organized layer of TS. Some particles are covered by stacks of more than one layer and, in that case, there is no curvature (Figures $5 b$ and $5 c$ ). Assuming that the TS stacks were oriented edge-on, an interlayer distance of about $4.5 \mathrm{~nm}$ was measured, which perfectly agrees with the value of the lamellar spacing in $\beta$-TS and the value measured from the AFM images of the SLN particles.

Several types of NLC particles prepared in the presence of PEG 660-stearate were also observed by cryo-TEM (Figures $5 \mathrm{~d}$ to $5 \mathrm{f}$ ). The particles have facetted irregular shapes and, again, seem to contain two regions with different lighter and darker contrasts (Figure 5d). As in the case of NLCs prepared with Poloxamer 188, some particles are irregularly covered with stacks of two to six $4.5 \mathrm{~nm}$-spaced dark layers that likely correspond to $\beta$-TS (Figures 5e and 5f). More rarely, particles with a more complex shape were observed. For instance, the spoon-like particle seen in Figure $5 \mathrm{~d}$ is constituted by a straight region and a curved region, both of which appear to be layered. 
(a)

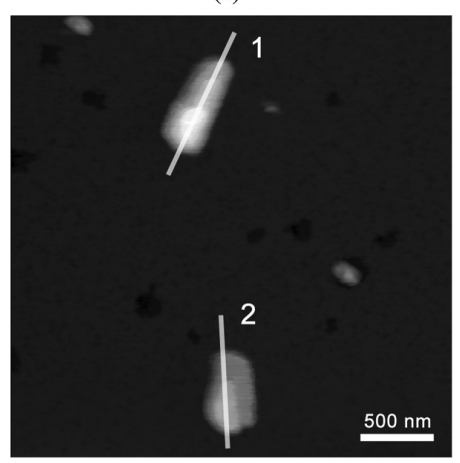

(b)

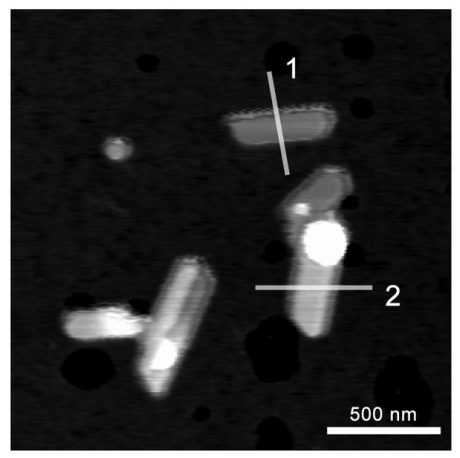

recorded from the freeze-dried specimens prepared with PEG 660-stearate exhibited the same peaks (Figure 6b), the peak distributions for the specimens prepared with Poloxamer 188 contained five peaks located at $2 \theta$ of 6 , 14.5, 18.5, 23 and $26^{\circ}$ (Figure 6 b). This profile corresponds to that of pure Poloxamer 188. Since Poloxamer 188 is in 5:1 proportion with respect to TS in the formulation, the peaks from the solid lipid were probably masked by those from the surfactant.

\section{Thermal properties}

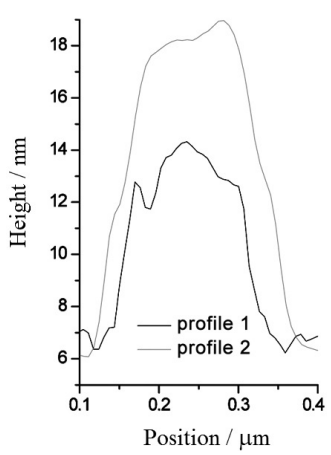

Figure 4. AFM images of SLN particles prepared with Polomaxer 188 (a) and PEG 660-stearate (b) as surfactants in the aqueous phase.

WAXS profiles of the samples were collected in order to determine the allomorphic form of TS (Figure 6). All concentrated suspensions (Figure 6a) yielded diffraction profiles that exhibited five main peaks at diffraction angles $2 \theta$ of $6,16.5,19,23$ and $24^{\circ}$, corresponding to lattice spacings of $1.47,0.54,0.46,0.39$ and $0.37 \mathrm{~nm}$, respectively. This peak distribution shows that TS is present in the sample in its $\beta$-form. ${ }^{10}$ While the diffraction profiles

DSC thermograms of castor oil and lecithin are shown in Figure 7a, while those of TS, Poloxamer 188 and PEG 660-stearate are shown in Figure 7b. The corresponding thermal events are summarized in Table 3. Pure lecithin, TS, Poloxamer 188 and PEG-stearate exhibited endothermic events at $75.9,73.6,54.3$ and $24.3{ }^{\circ} \mathrm{C}$, respectively.

Freeze-dried SLNs and NLCs prepared using PEG 660-stearate or Poloxamer 188 show two endothermic peaks (Figure 7c). In the samples prepared with PEG 660-stearate, the peaks are located at about 18 and $70{ }^{\circ} \mathrm{C}$. In the samples prepared with Poloxamer 188, the peaks are at about 51 and $70{ }^{\circ} \mathrm{C}$. In all four samples, the peaks at $70{ }^{\circ} \mathrm{C}$ were assigned to $\beta$-TS melting, although the temperature was slightly lower than that of pure TS $\left(73.6^{\circ} \mathrm{C}\right)$. The other peaks in each thermogram are related to the respective surfactant.

This slight reduction in the TS melting temperature may result from the small particle size of the lipid nanocarriers since lipid nanocrystals were found to melt at a temperature $3-5{ }^{\circ} \mathrm{C}$ lower than the corresponding bulk material. ${ }^{11} \mathrm{No}$ significant difference in the melting point was observed for
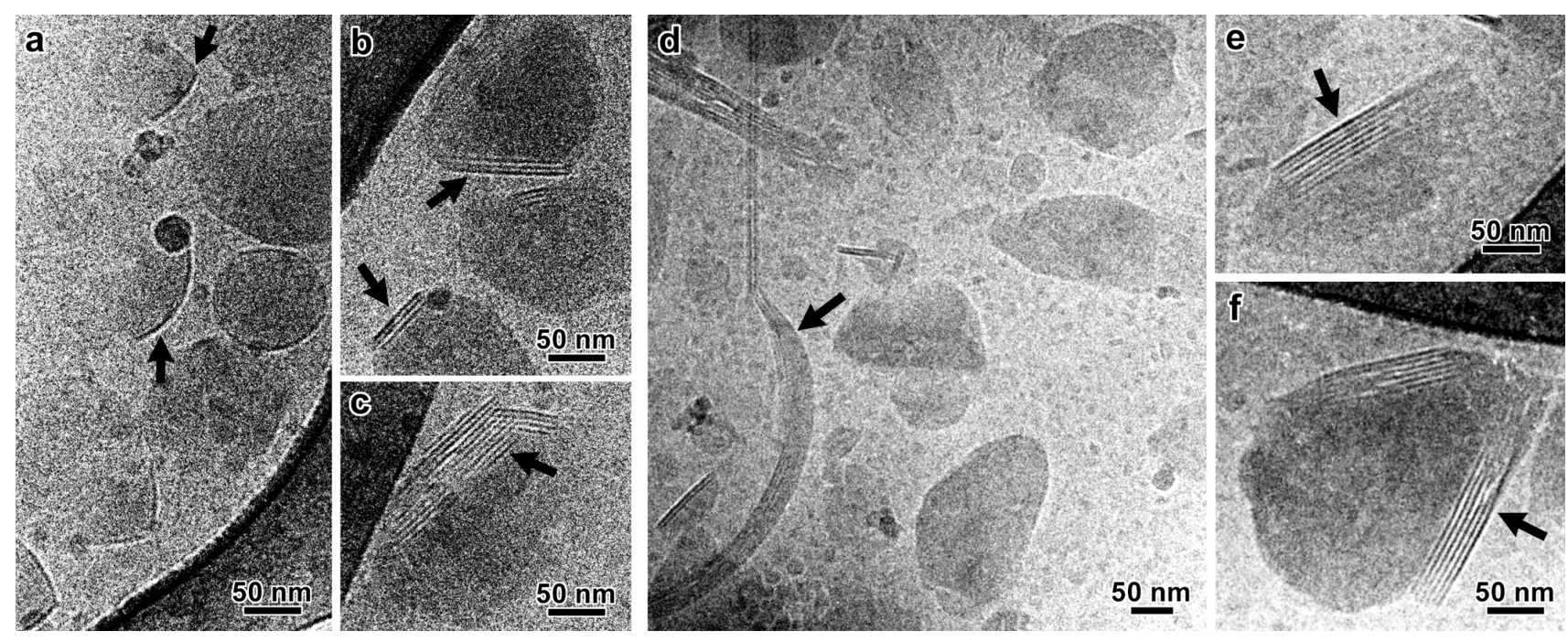

Figure 5. Cryo-TEM images of NLC suspensions prepared using Poloxamer 188 (a-c) and PEG 660-stearate (d-f) as surfactants in the aqueous phase. The arrows point to tristearin layers. 
TS when SLNs and NLCs were analyzed, indicating that the presence of CO did not induce any change in the lipid arrangement of the solid lipid.

\section{Discussion}

In the present study, three nanoparticulate systems (SLNs, NLCs and NEs) were investigated, and differences in their ultrastructure were evaluated. The lipid components (TS and $\mathrm{CO}$ ) display similar structures and both consist of

(a)

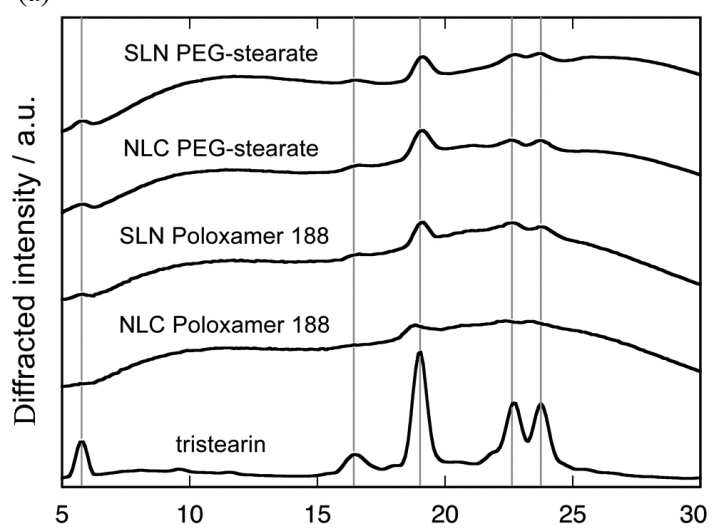

(b)

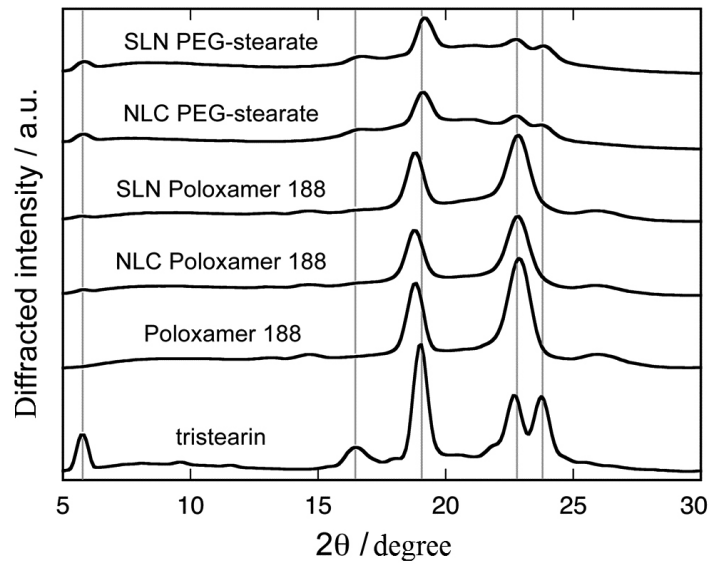

Figure 6. WAXS profiles of concentrated (a) and freeze-dried (b) lipidbased colloidal suspensions. The diffraction profile of tristearin was added as a reference.

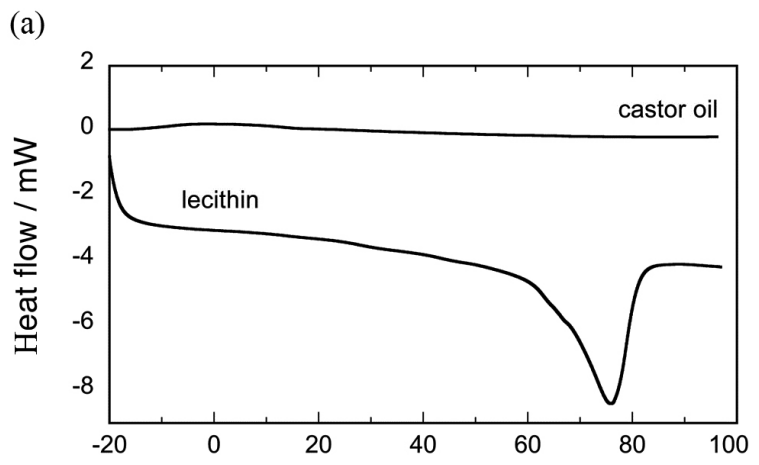

(b)

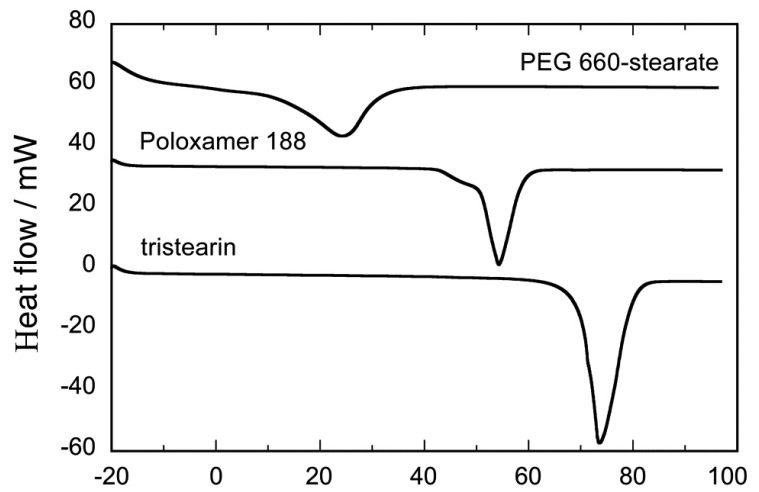

(c)

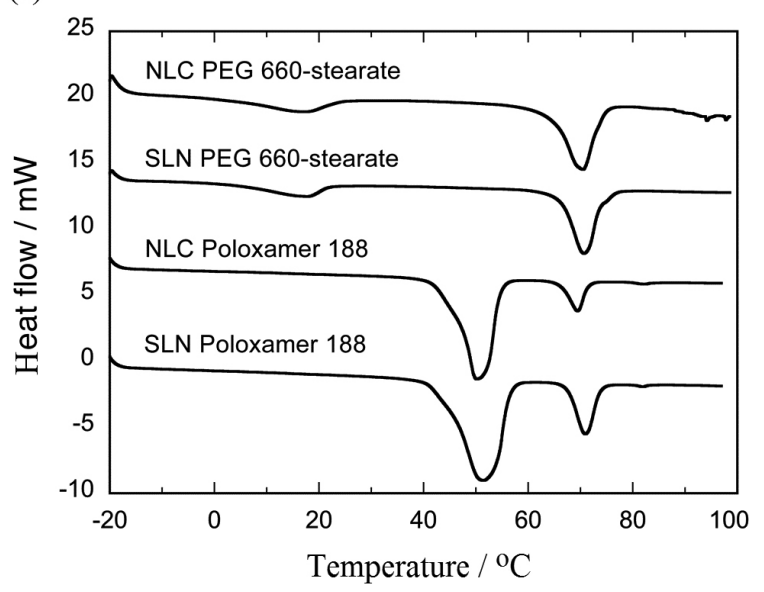

Figure 7. DSC thermograms of castor oil and lecithin (a), TS, Poloxamer 188 and PEG 660-stearate (b), and freeze-dried SLN and NLC particles prepared using PEG 660-stearate or Poloxamer 188 (c).

Table 3. Parameters obtained from DSC measurements of freeze-dried lipid-based colloids prepared with TS, Poloxamer 188 and PEG 660-stearate

\begin{tabular}{|c|c|c|c|c|c|c|c|c|}
\hline & \multicolumn{2}{|c|}{ Tristearin } & \multicolumn{2}{|c|}{ Poloxamer 188} & \multicolumn{2}{|c|}{ PEG 660-stearate } & \multicolumn{2}{|c|}{ Lecithin } \\
\hline & $\mathrm{T}_{\mathrm{m}} /{ }^{\circ} \mathrm{C}$ & $\Delta \mathrm{H} /\left(\mathrm{J} \mathrm{g} \mathrm{g}^{-1}\right)$ & $\mathrm{T}_{\mathrm{m}} /{ }^{\circ} \mathrm{C}$ & $\Delta \mathrm{H} /\left(\mathrm{J} \mathrm{g}^{-1}\right)$ & $\mathrm{T}_{\mathrm{m}} /{ }^{\circ} \mathrm{C}$ & $\Delta \mathrm{H} /\left(\mathrm{J} \mathrm{g} \mathrm{g}^{-1}\right)$ & $\mathrm{T}_{\mathrm{m}} /{ }^{\circ} \mathrm{C}$ & $\Delta \mathrm{H} /\left(\mathrm{J} \mathrm{g}^{-1}\right)$ \\
\hline Raw material & 73.6 & 207.6 & 54.3 & 129.0 & 24.3 & 78.3 & 75.9 & 47.1 \\
\hline $\operatorname{SLN}_{\text {Poloxamer } 188^{a}}$ & 70.9 & 159.5 & 51.4 & 111.5 & - & - & - & - \\
\hline $\mathrm{NLC}_{\text {Poloxamer } 188^{\mathrm{a}}}$ & 69.5 & 197.9 & 50.3 & 108.2 & - & - & - & - \\
\hline $\operatorname{SLN}_{\mathrm{PEG} 660 \text {-stearate }}{ }^{\mathrm{b}}$ & 70.7 & 122.4 & - & - & 17.9 & 56.1 & - & - \\
\hline $\mathrm{NLC}_{\mathrm{PEG}}$ 660-stearate & 70.5 & 146.3 & - & - & 18.2 & 29.8 & - & - \\
\hline
\end{tabular}

${ }^{a} \mathrm{SLN}_{\text {Poloxamer 188, }}, \mathrm{NLC}_{\text {Poloxamer 188: }}$ : colloidal suspensions prepared using Poloxamer 188 as hydrophilic surfactant; ${ }^{\mathrm{b}} \mathrm{SLN}_{\mathrm{PEG}}$ 660-stearate NLC $_{\mathrm{PEG}}$ 660-stearate: colloidal suspensions prepared using PEG 660-stearate as hydrophilic surfactant. $\mathrm{T}_{\mathrm{m}}$ : phase transition temperature. 
triglycerides containing 18-carbon fatty acid chains. On the one hand, as can be seen in Table 2, smaller particles were obtained when the liquid lipid alone was used in the formulation to obtain nanoemulsions, and no difference in the measured size was observed when analyses were carried out at angles of $90^{\circ}(\mathrm{AVL})$ and $173^{\circ}$ (Zetasizer). On the other hand, larger particles were obtained for SLNs and NLCs, and differences in the measured mean size were observed at different measurement angles. These results are probably related to the morphological features of SLNs and NLCs. The morphological evaluation clearly showed that platelet-shaped nanoparticles were obtained when the lipid consisted of only TS. In addition, as shown by TEM and cryo-TEM images, a mixture of CO and TS with an oil-load of $50 \%$ resulted in the formation of hybrid nanoparticles, in which phase separation occurred between the liquid and solid lipids after emulsion cooling and TS crystallization. DLS measures the diffusion coefficients of particles and, under the assumption of spherical particle shape, autocorrelation analysis yields a mean particle diameter. ${ }^{12,13}$ Therefore, when the nanocarriers are anisometric, the observed diameter could be overestimated because the hydrodynamic diameter is calculated based on an equivalent sphere with the same diffusion coefficient. As a result, the particle size measured from TEM images and by DLS should not directly be compared for SLN dispersions. The anisometric shapes of SLNs and NLCs may also have contributed to the discrepancies in the sizes obtained by DLS. Particles with different sizes scatter with different intensities depending on the scattering angle. At certain angles, the scattering intensity of the larger particles may completely overwhelm the weak scattering signal of the smaller particles, thus masking the data analysis at that angle. ${ }^{14-16}$ This could explain the low PDI value obtained for the NLC colloidal dispersions by DLS, in spite of the presence of a second particle size population that was visible by TEM and cryo-TEM images.

The differences found in the DLS mean sizes of SLN, NLC and NE by DLS were also reported by Jores et al. ${ }^{17}$ for lipid nanodispersions prepared by hot homogenization technique. These authors showed a decrease in the mean size of lipid nanodispersions based on the relative amounts of glyceryl behenate (GB) and medium chain triglycerides (MCT). Considering that MCT and melted GB are similar at temperature above $70{ }^{\circ} \mathrm{C}$, the authors postulated that the differences in the sizes resulted not from homogenization efficiency, but rather from the resulting shape of the nanoparticles following crystallization. Jores et al. ${ }^{17}$ also described the phase separation between caprylic/capric triglyceride and glyceryl behenate when an oil-load of $30 \%$ was used. This result was supported by fluorometric spectroscopy, which clearly demonstrated that NLC nanoparticles offered two compartments to accommodate Nile Red, a lipophilic fluorescent molecule. ${ }^{18}$ In our case, ricinoleic acid, the main constituent of castor oil, is a monounsaturated 18-carbon fatty acid, that has a hydroxyl functional group on the $12^{\text {th }}$ carbon. This functional group makes castor oil a more polar structure, which may have contributed to the segregation of the solid and liquid lipids in the nanoparticle structure.

Polymorphism is one of the most important features that affect the stability of solid dosage forms because, even though they are chemically identical, polymorphs generally have different thermal properties (melting temperature), crystal structures and solubility. TS crystallizes into four different crystalline forms, namely the $\gamma, \alpha, \beta^{\prime}$ and $\beta$ forms, and their formation mainly depends on the cooling and stirring rates. ${ }^{6}$ The $\alpha$-form tends to quickly transform into the $\beta^{\prime}$-form, which has a tighter chain packing. The $\beta$-form is the most stable; therefore, the transition of liquid (melt) from $\alpha$ to $\beta$ via $\beta$ ' is the pathway followed by triglycerides to achieve an optimum molecular packing. Bunjes et al. ${ }^{7}$ investigated TS polymorphism in solid lipid nanoparticles stabilized by a blend of saturated long-chain phospholipids and the bile salt sodium glycolate. The structures of the dispersions were investigated by DSC, WAXS and TEM. A platelet-like, layered structure was confirmed for particles in the $\beta$-modification, whereas a spheroidal shape was revealed for particles in the $\alpha$-form. In our study, the morphological evaluation as well as the WAXS and DSC analyses indicated the formation of $\beta$-TS in SLNs. This result may be attributed to the preparation method. By using the hot solvent diffusion method to obtain the lipid nanocarriers, hot nanosized emulsions were generated by mixing the organic and aqueous phases at $10{ }^{\circ} \mathrm{C}$, the melting temperature of the solid lipid and, subsequently, magnetically stirring the mixture until it cooled to room temperature. This procedure could have favored the formation of the $\beta$ allomorph because the recrystallization step was slow.

In a previous study, our group demonstrated that an increase in $\mathrm{CO}$ content in the formulation led to an increase in the QU loading in the lipid nanocarriers. However, the increase in $\mathrm{CO}$ content did not affect the quercetin release rate. For both QU-loaded SLN (TS:CO 100:0) and QU-NLC (TS:CO 50:50), QU release was about 45 and $70 \%$ when Poloxamer 188 and PEG-stearate were used as the hydrophilic surfactant, respectively. ${ }^{5}$ Jenning et al. ${ }^{19}$ also demonstrated that mixing liquid and solid lipids circumvents the limited drug loads of conventional SLNs. However, in contrast to the results obtained by these authors, our study did not reveal the formation of lipid 
nanocarriers containing liquid nanocompartments. Our results are more in agreement with those obtained by Hu et al..$^{20}$, who demonstrated that the liquid lipid was not located inside of the particles. In our case, NLCs displayed distinct compartments, in which an oil compartment increased the incorporation capacity of the system due to the ability of the oily lipid to dissolve a hydrophobic drug. Further, no retardation of the drug release occurred because the castor oil was not located in an inner core surrounded by solid lipid. Therefore, NLCs constituted of TS and CO do not seem to be advantageous for carrying hydrophobic drugs such as quercetin.

\section{Conclusions}

The results of the present work demonstrated that lipid nanodispersions made from glyceryl tristearate and/or castor oil display different sizes and structures. While the nanoemulsions exhibited spherical structure, SLNs and NLCs presented more complex shapes. SLNs appeared to be lamellar crystals while NLCs exhibited a hybrid structure composed of a lamellar crystalline phase (assigned to tristerarin in the $\beta$-form) in contact with a liquid oil compartment. Although previous studies showed that an increase in castor oil load in the nanoparticles caused an increase in drug loading, advantages related to the retardation of the drug release cannot be expected for these drug delivery systems since the liquid and solid lipids are separated in distinct compartments.

\section{Acknowledgements}

C. L. Dora benefited from a PhD grant from Coordenação de Aperfeiçoamento de Pessoal de Nível Superior (CAPES).

\section{References}

1. Bunjes, H.; Westensen, K. In Crystallization Processes in Fats and Lipid Systems; Garti, N.; Sato, K., eds.; Marcel Dekker: New York, NY, USA, 2001, chapter 12.

2. Li, H.; Zhao, X.; Ma, Y.; Zhai, G.; Li, L.; Lou, H.; J. Controlled Release 2009, 10, 238.
3. Saupe, A.; Gordon, K.; Rades, T.; Int. J. Pharm. 2006, 314, 56.

4. Chakraborty, S.; Shukla, D.; Mishra, B.; Singh, S.; Eur. J. Pharm. Biopharm. 2009, 73, 1.

5. Dora, C. L.; Silva, L. F. C.; Tagliari, M. P.; Silva, M. A. S.; Lemos-Senna, E.; Lat. Am. J. Pharm. 2011, 30, 289.

6. Hartel, R. Crystallization in Foods; Aspen Publishers: Gaithersburg, MD, USA, 2001.

7. Bunjes, H.; Steiniger, F.; Richter, W.; Langmuir 2007, 23, 4005.

8. Kawashima, Y.; Yamamoto, H.; Takeuchi, H.; Hino, T.; Niwa, T.; Eur. J. Pharm. Biopharm. 1998, 45, 41.

9. Hu, F. Q.; Yuan, H.; Zhang, H.; Fang, M.; Int. J. Pharm. 2002, 239, 121.

10. Oh, J.; McCurdy, A.; Clark, S.; Swanson, B.; Food Chem. Toxicol. 2002, 67, 2911.

11. Heurtault, B.; Saulnier, P.; Pech, B.; Proust, J.; Benoit, J.; Biomaterials 2003, 24, 4283.

12. Haskell, R.J.; Shifflet, R. J.; Elzinga, P. A. In Submicron Emulsions in Drug Targeting and delivery; Benita, S., ed.; Hardwood Academic Publishers: Amsterdam, The Netherlands, 1998, chapter 3.

13. Bunjes, H.; Koch, M. H. J.; Westesen, K.; Langmuir 2000, 16, 5234.

14. Cantor, C. R.; Schimmel, P. R.; Biophysical Chemistry, Part II: Techniques for the Study of Biological Structure and Function; Freeman and Co.: Oxford, UK, 1980.

15. Sjöström, B.; Kaplun, A.; Talmon, Y.; Cabane, B.; Pharm. Res.1995, 12, 39.

16. Dickinson, E.; Miller, R.; Westesen, K.; Drechsler, M.; Bunjes, H. In Food Colloids: Fundamentals of Formulation; Dickinson, E.; Miller, R., eds.; Royal Society of Chemistry: Cambridge, UK, 2001.

17. Jores, K.; Mehnert, W.; Drechsler, M.; Bunjes, H.; Johann, C.; Mäder, K.; J. Controlled Release 2004, 95, 217.

18. Jores, K.; Haberland, A.; Wartewig, S.; Mäder, K.; Mehnert, W.; Pharm. Res. 2005, 22, 1887.

19. Jenning, V.; Thünemann, A. F.; Gohla, S. H.; Int. J. Pharm. 2000, 199, 167.

20. Hu, F. Q.; Jiang, S. P.; Du, Y. Z.; Yuan, H.; Ye, Y. Q.; Zeng, S.; Colloids Surf., B 2005, 45, 167.

Submitted: June 6, 2012 Published online: October 30, 2012 